\title{
EIGENVALUES OF SOME ALMOST PERIODIC FUNCTIONS
}

\author{
JIRO EGAWA
}

(Communicated by Dennis Burke)

\begin{abstract}
Let $B_{U}$ be the set of real valued functions on $R$ which are bounded and uniformly continuous. For $f, g \in B_{U}$, put

$$
d(f, g)=\sup _{t \in R}|f(t)-g(t)| \text {. }
$$

Then $B_{U}$ becomes a metric space. On $B_{U}$ we define a flow $\eta$ by $\eta(f, t)=f_{t}$ for $(f, t) \in B_{U} \times R$. We denote the restriction of $\eta$ to the hull of $f \in B_{U}$ by $\eta_{f}$. If $f$ is almost periodic, then the set of eigenvalues of $\eta_{f}$ coincides with the module of $f$ (see J. Egawa, Eigenvalues of compact minimal flows, Math. Seminar Notes (Kobe Univ.), 10 (1982), 281-291. In this paper, we extend this result to almost periodic functions with some additional properties.
\end{abstract}

We denote the sets of real numbers and complex numbers by $R$ and $C$, respectively. Let $X$ be a metric space with metric $d_{X}$. A continuous mapping $T: X \times R \rightarrow X$ is called a flow on (a phase space) $X$, if $T$ satisfies the following two conditions:

(1) $T(x, 0)=x$ for $x \in X$.

(2) $T(T(x, t), s)=T(x, t+s)$ for $x \in X$ and $t, s \in R$.

The orbit of $T$ through $x \in X$ is denoted by $C_{T}(x)$, that is, $C_{T}(x)=$ $\{T(x, t) ; t \in R\}$. The closure of a set $A \subset X$ is denoted by $\bar{A}$. A subset $M \subset X$ is called an invariant set of $T$ if we have $C_{T}(x) \subset M$ for every $x \in M$. We denote the restriction of $T$ to an invariant set $M$ of $T$ by $T \mid M$. A nonempty compact invariant set $M$ of $T$ is called a minimal set of $T$ if we have $\overline{C_{T}(x)}=M$ for every $x \in M$. If $X$ is itself a minimal set of $T$, we say that $T$ is a minimal flow on $X$. We say that $T$ is equicontinuous, if for every $\varepsilon>0$ there exists a $\delta>0$ such that for $x, y \in X$ with $d_{X}(x, y)<\delta$ and $t \in R$ we have $d_{X}(T(x, t), T(y, t))<\varepsilon$.

Proposition 1. Let $T$ be a flow on a compact metric space $X$. If $T$ is equicontinuous, then for every $x \in X \overline{C_{T}(x)}$ is a minimal set of $T$.

Proof. Easy.

Let $T_{n}$ be flows on $X_{n} \quad(n=1,2, \ldots)$. We denote the product flow of $\left\{T_{n}\right\}$ on $\prod_{n=1}^{\infty} X_{n}$ by $\prod_{n=1}^{\infty} T_{n}$.

Received by the editors June 8, 1990 and, in revised form, December 3, 1990.

1980 Mathematics Subject Classification (1985 Revision). Primary 54H20.

Key words and phrases. Equicontinuous, minimal flow, almost periodic function, eigenvalues. 
Proposition 2. Let $T_{n}$ be flows on compact metric spaces $X_{n}(n=1,2, \ldots)$. If for every $n T_{n}$ is equicontinuous, then the flow $\prod_{n=1}^{\infty} T_{n}$ is also equicontinuous. Proof. See [2, p. 27].

Let $T$ be a minimal flow on a compact metric space $X . \alpha \in R$ is called an eigenvalue of $T$ if there exists a continuous function $\chi_{\alpha}: X \rightarrow K$ such that $\chi_{\alpha}(T(x, t))=\chi_{\alpha}(x) \exp (2 \pi i \alpha t)$ for $(x, t) \in X \times R$, where $K$ is the unit circle in the complex plane. In this case $\chi_{\alpha}$ is called an eigenfunction of $T$ belonging to $\alpha$. We denote the set of eigenvalues of $T$ by $\Lambda(T)$. We can see that $\Lambda(T)$ is an additive subgroup of $R$.

Proposition 3. Let $T$ be an equicontinuous minimal flow on a compact metric space $X$. Then for each pair of distinct points $x, y \in X$ there exists $\alpha \in \Lambda(T)$ such that $\chi_{\alpha}(x) \neq \chi_{\alpha}(y)$.

Proof. By equicontinuity of $T$, we can define a group structure on $X$, and by this structure $X$ becomes an Abelian topological group [7, p. 101]. We can easily see that every character of $X$ with respect to this group structure is an eigenfunction of $T$. It follows that the proposition holds [6].

For the set $A \subset R$, we denote the least additive subgroup of $R$ containing $A$ by $\tilde{A}$.

Proposition 4. Let $T$ be an equicontinuous minimal flow on a compact metric space $X$, and $A \subset \Lambda(T)$. If for each pair of distinct points $x, y \in X$ there exists $\alpha \in A$ such that $\chi_{\alpha}(x) \neq \chi_{\alpha}(y)$, then we have $\tilde{A}=\Lambda(T)$.

Proof. Since $\Lambda(T)$ is a subgroup of $R$, we have $\tilde{A} \subset \Lambda(T)$. Let $C(X)$ be the set of complex valued continuous functions on $X$ with the topology of uniform convergence, and $O \subset C(X)$ all of linear combinations of $\left\{\chi_{\alpha}\right\}_{\alpha \in \tilde{A}}$. Then $O$ is obviously a linear subspace of $C(X)$. Since for $\alpha, \beta \in \widetilde{A}$ we have $\chi_{\alpha} \cdot \chi_{\beta}=\chi_{\alpha+\beta}$, and we have $f \cdot g \in O$ for $f, g \in O$. Further, every constant function belongs to $O$. If $f \in O$, then $\bar{f} \in O$, because for every $\alpha \in \widetilde{A}$ we have $\bar{\chi}_{\alpha}=\chi_{-\alpha}$, where $\bar{f}$ is a complex conjugate of $f$. Hence by the assumption and Stone-Weierstrass' theorem [5, p. 119], $O$ is dense in $C(X)$. Let $\mu$ be a unique invariant Borel measure of $T$ (since $T$ is equicontinuous, $T$ is strictly ergodic $[4$, p. 510$])$, and $L^{2}(X, \mu)$ the set of square summable complex valued functions. We can easily see that

$$
\left(\chi_{\alpha}, \chi_{\beta}\right)=\int_{X} \chi_{\alpha}(x) \overline{\chi_{\beta}(x)} d \mu(x)=0
$$

for $\alpha, \beta \in \Lambda(T) \quad(\alpha \neq \beta)$. Let $\beta \in \Lambda(T)-\tilde{A}$. Then there exists a sequence $\left\{h_{n}\right\} \subset 0$ such that $h_{n} \rightarrow \chi_{\beta}$ uniformly as $n \rightarrow \infty$. Since $\left(\chi_{\beta}, h_{n}\right)=0$ for every $n$ by the above remark, we obtain $\lim _{n \rightarrow \infty}\left(\chi_{\beta}, h_{n}\right)=\left(\chi_{\beta}, \chi_{\beta}\right)=0$. This means $\chi_{\beta} \equiv 0$. This is a contradiction. Hence we have $\tilde{A}=\Lambda(T)$.

Proposition 5. Let $T_{n}$ be equicontinuous minimal flows on compact metric spaces $X_{n}(n=1,2, \ldots)$. Put $X=\prod_{n=1}^{\infty} X_{n}$ and $T=\prod_{n=1}^{\infty} T_{n}$. Let $x \in X$, $M=\overline{C_{T}(x)}$, and $A=\bigcup_{n=1}^{\infty} \Lambda\left(T_{n}\right)$. Then we have $\tilde{A}=\Lambda(T \mid M)$.

Proof. By Propositions 1 and 2, $M$ is a minimal set of $T$ and $T \mid M$ is equicontinuous. Let $\alpha \in \Lambda\left(T_{n}\right)$. We denote the eigenfunction of $T_{n}$ belonging to $\alpha$ by 
$\chi_{\alpha}^{(n)}$. Define a function $\chi_{\alpha}: M \rightarrow K$ by $\chi_{\alpha}(x)=\chi_{\alpha}^{(n)}\left(x_{n}\right)$, where $x_{n}$ is the $n$th coordinate of $x$. Then $\chi_{\alpha}$ is continuous, and it is an eigenfunction of $T \mid M$ belonging to $\alpha$. Hence $A \subset \Lambda(T \mid M)$. Let $x, y \in M(x \neq y)$. Then there exists $n$ such that $x_{n} \neq y_{n}$. By Proposition 3 there exists $\alpha \in \Lambda\left(T_{n}\right)$ such that $\chi_{\alpha}^{(n)}\left(x_{n}\right) \neq \chi_{\alpha}^{(n)}\left(y_{n}\right)$. Hence by Proposition 4 we have $\widetilde{A}=\Lambda(T \mid M)$.

We denote the $n$-dimensional Euclidean space by $R^{n}$. Let $B_{U}^{(n)}$ be the set of $R^{n}$-valued continuous functions on $R$ which are bounded and uniformly continuous. For $f, g \in B_{U}^{(n)}$, put $d(f, g)=\sup _{t \in R}|f(t)-g(t)|$. Then $B_{U}^{(n)}$ becomes a metric space by this metric. On $B_{U}^{(n)}$ we define a flow $\eta$ by $\eta(f, t)=$ $f_{t}$ for $(f, t) \in B_{U}^{(n)} \times R$, where $f_{t}(s)=f(t+s)$ for $s \in R$. Then $\eta$ is obviously equicontinuous. For $f \in B_{U}^{(n)}$, put $H(f)=\overline{C_{\eta}(f)}=\overline{\left\{f_{t}\right\}_{t \in R}}$, and we denote the restriction of $\eta$ to $H(f)$ by $\eta_{f}$. A $R^{n}$-valued continuous function $f$ on $R$ is said to be almost periodic if for each $\varepsilon>0$ there exists a relatively dense subset $A_{\varepsilon}$ of $R$ such that $|f(t+\tau)-f(t)|<\varepsilon$ for $t \in R$ and $\tau \in A_{\varepsilon}$. We denote the set of $R^{n}$-valued almost periodic functions on $R$ by $A P^{(n)}$. Then the following proposition is known [3].

Proposition 6. Let $f \in A P^{(n)}$. Then

(1) $f \in B_{U}^{(n)}$.

(2) $H(f)$ is compact, and hence $\eta_{f}$ is an equicontinuous minimal flow on $H(f)$.

(3) For each $\alpha \in R, \lim _{t \rightarrow \infty} \frac{1}{t} \int_{0}^{t} f(s) \exp (-2 \pi i \alpha s) d s$ exists.

For $f \in A P^{(n)}$, put $\Lambda_{f}=\left\{\alpha \in R ; \lim _{t \rightarrow \infty} \frac{1}{t} \int_{0}^{t} f(s) \exp (-2 \pi i \alpha s) d s \neq 0\right\}$.

Proposition 7. Let $f \in A P^{(n)}$. Then $\tilde{\Lambda}_{f}=\Lambda\left(\eta_{f}\right)$.

Proof. We sketch the proof for $n=1$ (see [1] for detail). Define a function $F: H(f) \rightarrow R$ by $F(g)=g(0)$ for $g \in H(f)$. Then $F$ is continuous on $H(f)$ and $F\left(\eta_{f}(g, t)\right)=F\left(g_{t}\right)=g_{t}(0)=g(t)$ for $(g, t) \in H(f) \times R$. Let $\Delta$ be a set of eigenfunctions of $\eta_{f}$. Then $\Delta$ coincides with the set of characters of the associated topological group $H(f)$. Let $\lambda \notin \Lambda\left(\eta_{f}\right)$, and $\varepsilon>0$. Then there exist $\left\{a_{j}\right\}_{j=1}^{N} \subset C$ and $\left\{\chi_{\lambda_{j}}\right\} \subset \Delta$ such that

$$
\left|F(g)-\sum_{j=1}^{N} a_{j} \chi_{\lambda_{j}}(g)\right|<\varepsilon
$$

for $g \in H(f)$ [6]. Since

$$
\frac{1}{t} \int_{0}^{t}\left|F\left(f_{s}\right)-\sum_{j=1}^{N} a_{j} \chi_{\lambda_{j}}\left(f_{s}\right)\right| d s<\varepsilon
$$

for every $t \in R$, we have

$$
\varepsilon \geq\left|\lim _{t \rightarrow \infty} \frac{1}{t} \int_{0}^{t} f(s) \exp (-2 \pi i \lambda s) d s\right|,
$$

because $\lambda \neq \lambda_{j}(j=1,2, \ldots, N)$ and

$$
\lim _{t \rightarrow \infty} \frac{1}{t} \int_{0}^{t} \exp \left(-2 \pi i\left(\lambda-\lambda_{j}\right)\right) d s=0 .
$$


Hence, since $\varepsilon>0$ is arbitrary, we have

$$
\lim _{t \rightarrow \infty} \frac{1}{t} \int_{0}^{t} f(s) \exp (-2 \pi i \lambda s) d s=0 .
$$

This implies $\lambda \notin \Lambda_{f}$, which implies $\Lambda_{f} \subset \Lambda\left(\eta_{f}\right)$. Further, we shall show $\Lambda_{f}$ satisfies the condition of Proposition 4. Let $\Lambda_{f}=\left\{\lambda_{i_{j}}\right\} \subset \Lambda\left(\eta_{f}\right)$ and $\chi_{i_{j}}$ be an eigenfunction of $\eta_{f}$ belonging to $\lambda_{i_{j}}$. Then there exists a sequence $\left\{f_{n}\right\}$ such that

$$
f_{n}(t)=\sum_{j=1}^{l_{n}} a_{j}^{(n)} \exp \left(2 \pi i \lambda_{i_{j}} t\right) \quad\left(a_{j}^{(n)} \in C\right)
$$

converges to $f$ uniformly on $R$ as $n \rightarrow \infty$ [3, p. 48]. Put

$$
F_{n}(g)=\sum_{j=1}^{l_{n}} a_{j}^{(n)} \chi_{i_{j}}^{-1}(f) \chi_{i_{j}}(g)
$$

for $g \in H(f)$. Then $F_{n}$ is continuous on $H(f)$, and

$$
\left|F_{n}\left(f_{t}\right)-F\left(f_{t}\right)\right|=\left|f^{(n)}(t)-f(t)\right|
$$

for $t \in R$. Since $\left\{f_{t}\right\}_{t \in R}$ is dense in $H(f),\left\{F_{n}\right\}$ converges to $F$ uniformly on $H(f)$. We assume that $\chi_{i_{j}}(g)=\chi_{i_{j}}(h)(g, h \in H(f))$ for all $j$. Then we have $g(t)=F\left(g_{t}\right)=\lim _{n \rightarrow \infty} F_{n}\left(g_{t}\right)=\lim _{n \rightarrow \infty} F_{n}\left(h_{t}\right)=F\left(h_{t}\right)=h(t)$ for $t \in R$. Hence $g=h$, which implies that $\Lambda_{f}$ satisfies the condition of Proposition 4. Hence we have $\widetilde{\Lambda}_{f}=\Lambda\left(\eta_{f}\right)$.

For $n \geq 2$ we can easily prove the proposition for considering product flows.

Proposition 8. Let $A_{n} \subset R \quad(n=1,2, \ldots), A=\bigcup_{n=1}^{\infty} A_{n}$, and $B=\bigcup_{n=1}^{\infty} \widetilde{A}_{n}$. Then we have $\widetilde{A}=\widetilde{B}$.

Proof. Easy.

Let $T$ and $S$ be flows on $X$ and $Y$, respectively. A continuous mapping $h: X \rightarrow Y$ is called a homomorphism from $T$ to $S$ if $H(T(x, t))=S(h(x), t)$ holds for $(x, t) \in X \times R$. Further, if $h$ is a homeomorphism from $X$ onto $Y$, then we say that $h$ is an isomorphism from $T$ to $S$.

Proposition 9. Let $T$ and $S$ be minimal flows on compact metric spaces $X$ and $Y$, respectively. If there exists an isomorphism from $T$ to $S$, then we have $\Lambda(T)=\Lambda(S)$.

Proof. Easy.

Let $W \subset R^{n}$ be an open set, and $B U^{(n)}(W)$ the set of continuous functions from $W \times R$ to $R^{n}$ which satisfy the following condition: $f \in B U^{(n)}(W)$ if and only if for each compact set $K \subset W \quad f$ is bounded and uniformly continuous on $K \times R$. We define a metric on $B U^{(n)}(W)$ in the following way. Let $\left\{K_{m}\right\}_{m=1}^{\infty}$ be a sequence of compact subsets of $W$ such that $K_{m} \subset K_{m+1}$ $(m=1,2, \ldots)$ and $W=\bigcup_{m=1}^{\infty} K_{m}$. For $f, g \in B U^{(n)}(W)$, put

$$
\begin{aligned}
& d_{m}(f, g)=\sup _{(x, t) \in K_{m} \times R}\{|f(x, t)-g(x, t)|\}, \\
& \rho_{m}(f, g)=\frac{d_{m}(f, g)}{1+d_{m}(f, g)}
\end{aligned}
$$


and

$$
\rho(f, g)=\sum_{n=1}^{\infty} \frac{1}{2^{n}} \rho_{n}(f, g) .
$$

Then $B U^{(n)}(W)$ becomes a metric space by this $\rho$. On $B U^{(n)}(W)$ we define a flow $\xi$ by $\xi(f, t)=f_{t}$ for $(f, t) \in B U^{(n)}(W) \times R$, where $f_{t}(x, s)=f(x, t+s)$ for $(x, s) \in W \times R$. It is easy to verify that it is well defined. Obviously $\xi$ is an equicontinuous flow on $B U^{(n)}(W)$. For $f \in B U^{(n)}(W)$, put $\Omega(f)=\overline{C_{\xi}(f)}=$ ${\overline{\left\{f_{t}\right\}_{t \in R}}}_{t}$ and $\xi_{f}=\xi \mid \Omega(f)$. A continuous function $f: W \times R \rightarrow R^{n}$ is said to be almost periodic in $t$ uniformly for $x \in W$ if for each compact set $K \subset W$ and $\varepsilon>0$ there exists a relatively dense subset $A_{K \varepsilon}$ of $R$ such that $\tau \in A_{K \varepsilon}$ and $(x, t) \in K \times R$ imply $|f(x, t+\tau)-f(x, t)|<\varepsilon$. The set of continuous functions which are almost periodic in $t$ uniformly for $x \in W$ is denoted by $A P^{(n)}(W)$. The following proposition is known [3].

Proposition 10. Let $f \in A P^{(n)}(W)$. Then

(1) $f \in B U^{(n)}(W)$.

(2) $\Omega(f)$ is compact, and hence $\xi_{f}$ is an equicontinuous minimal flow on $\Omega(f)$.

(3) for $x \in W$ and $\alpha \in R$,

$$
\lambda(\alpha, x)=\lim _{t \rightarrow \infty} \frac{1}{t} \int_{0}^{t} f(x, s) \exp (-2 \pi i \alpha s) d s
$$

exists and $\lambda(\alpha, \cdot)$ is continuous on $W$.

For $f \in A P^{(n)}(W)$, put $\Lambda_{f}(W)=\{\alpha \in R ; \lambda(\alpha, x) \not \equiv 0\}$. Then we have the following theorem, which is the main theorem in this paper.

Theorem. Let $f \in A P^{(n)}(W)$. Then we have $\tilde{\Lambda}_{f}(W)=\Lambda\left(\xi_{f}\right)$.

Proof. Let $a \in W$, and $k_{a}(t)=f(a, t)$ for $t \in R$. Then $k_{a} \in A P^{(n)}$ and $H\left(k_{a}\right)=\{g(a, \cdot) ; g \in \Omega(f)\}$. Put $H\left(k_{a}\right)=H_{a}(f)$. Define a mapping $h_{a}$ from $\Omega(f)$ to $H_{a}(f)$ by $h_{a}(g)=g(a, \cdot)$ for $g \in \Omega(f)$. Then $h_{a}$ is obviously continuous and a homomorphism from $\xi_{f}$ to $\eta \mid H_{a}(f)$. Let $\left\{a_{m}\right\}_{m=1}^{\infty}$ be a dense subset of $W$. Put $Y=\prod_{n=1}^{\infty} H_{a_{m}}(f)$ and $T=\prod_{m=1}^{\infty} \eta \mid H_{a_{m}}(f)$. Then $T$ is an equicontinuous flow on the compact metric space $Y$ by Proposition 2, because $\eta \mid H_{a_{m}}(f)$ is equicontinuous for every $n$. Define a mapping $h$ from $\Omega(f)$ to $Y$ by $h(g)=\left(h_{a_{m}}(g)\right)$ for $g \in \Omega(f)$. Then $h$ is continuous and a homomorphism from $\xi_{f}$ to $T$. Further, we can easily see that $h$ is injection. Put $M=h(\Omega(f))$. Then we have $M=\overline{C_{T}(h(f))}$. Hence we have $\Lambda(T \mid M)=\tilde{A}$ by Proposition 5, where $A=\bigcup_{m=1}^{\infty} \Lambda\left(\eta \mid H_{a_{m}}(f)\right)$. Put $A_{m}=\left\{\alpha \in R ; \lim _{t \rightarrow \infty} \frac{1}{t} \int_{0}^{t} f\left(a_{m}, s\right) \exp (-2 \pi i \alpha s) d s \neq 0\right\}$. Then $\Lambda_{f}(W)=$ $\bigcup_{m=1}^{\infty} A_{m}$. In fact, $\bigcup_{m=1}^{\infty} A_{m} \subset \Lambda_{f}(W)$ is obvious. Let $\alpha \notin \bigcup_{m=1}^{\infty} A_{m}$. Then $\lambda\left(\alpha, a_{m}\right)=0$ for each $m$. Since $\lambda(\alpha, \cdot)$ is continuous on $W$ and $\left\{a_{m}\right\}_{m=1}^{\infty}$ is dense in $W$, we have $\lambda(\alpha, x) \equiv 0$. This means that $\alpha \notin \Lambda_{f}(W)$, which implies $\Lambda_{f}(W)=\bigcup_{m=1}^{\infty} A_{m}$. Since $\Lambda\left(\eta \mid H_{a_{m}}(f)\right)=\tilde{A}_{m}$ by Proposition 7 , we have $\Lambda(T \mid M)=\tilde{\Lambda}_{f}(W)$ by Proposition 8. Since $\xi_{f}$ is isomorphic to $T \mid M$, we obtain $\Lambda\left(\xi_{f}\right)=\Lambda(T \mid M)=\tilde{\Lambda}_{f}(W)$ by Proposition 9 . 
Corollary. Let $f, g \in A P^{(n)}(W)$. Then $\xi_{f}$ and $\xi_{g}$ are isomorphic if and only if $\tilde{\Lambda}_{f}(W)=\tilde{\Lambda}_{g}(W)$.

Proof. Since $\Lambda\left(\xi_{f}\right)=\tilde{\Lambda}_{f}(W)=\tilde{\Lambda}_{g}(W)=\Lambda\left(\xi_{g}\right)$ by the theorem and $\xi_{f}$ and $\xi_{g}$ are equicontinuous, the corollary follows.

\section{REFERENCES}

1. J. Egawa, Eigenvalues of compact minimal flows, Math. Seminar Notes (Kobe Univ.) 10 (1982), 281-291.

2. R. Ellis, Lectures on topological dynamics, Benjamin, New York, 1969.

3. A. M. Fink, Almost periodic differential equations, Lecture Notes in Math., vol. 377, Springer-Verlag, New York, 1974.

4. V. V. Nemytskii and V. V. Stepanov, Qualitative theory of differential equations, Princeton Univ. Press, Princeton, NJ, 1960.

5. M. Nibu, Topological groups, Iwanami, Tokyo, 1976. (Japanese)

6. L. S. Pontryagin, Topological groups, (Japanese Transl.) Iwanami, Tokyo, 1957.

7. G. R. Sell, Topological dynamics and ordinary differential equations, Van Nostrand Math. Studies, no. 33, Von Nostrand Reinhold, London, 1971.

Department of Mathematics, College of Liberal Arts, Kobe University, Nada, Kobe 657, JAPAN 